\title{
Subcellular Targeting of the Euplotes raikovi Kinase Er-MAPK1, as Revealed by Expression in Different Cell Systems
}

\author{
Annalisa Candelori', Takaharu G. Yamamoto ${ }^{2}$, Masaaki Iwamoto ${ }^{2}$, Maura Montani ${ }^{1}$, \\ Augusto Amici ${ }^{1}$ and Adriana Vallesi ${ }^{1 *}$
}

${ }^{1}$ School of Biosciences and Veterinary Medicine, University of Camerino, Camerino, Italy, ${ }^{2}$ Advanced ICT Research Institute, National Institute of Information and Communications Technology, Kobe, Japan

OPEN ACCESS

Edited by:

Judith Van Houten,

The University of Vermont,

United States

Reviewed by:

Eduardo Villalobo,

University of Seville, Spain

Helena Antunes Soares,

University of Lisbon, Portugal

Bruno Carmona

University of Lisbon, Portugal

${ }^{*}$ Correspondence:

Adriana Vallesi

adriana.vallesi@unicam.it

Specialty section:

This article was submitted to

Cell Growth and Division,

a section of the journal

Frontiers in Cell and Developmental

Biology

Received: 24 June 2019 Accepted: 04 October 2019

Published: 17 October 2019

Citation:

Candelori A, Yamamoto TG, Iwamoto M, Montani M, Amici A and Vallesi A (2019) Subcellular Targeting

of the Euplotes raikovi Kinase

Er-MAPK1, as Revealed by

Expression in Different Cell Systems.

Front. Cell Dev. Biol. 7:244.

doi: 10.3389/fcell.2019.00244
In the ciliate Euplotes raikovi, a 631-amino acid Er-MAPK1 protein kinase was found to localize in nucleoli of the transcriptionally active nucleus (macronucleus) and act as a key component of an autocrine, cell-growth promoting self-signaling mechanism. While its 283-amino acid N-terminal domain includes all the structural specificities of the mitogen-activated protein kinases required for a catalytic function, the 348amino acid C-terminal domain is structurally unique with undetermined functions. By expressing the two Er-MAPK1 domains tagged with the green fluorescent protein in mammalian fibroblasts, the yeast Schizosaccharomyces pombe and the ciliate Tetrahymena thermophila, evidence was obtained that the C-terminal domain contains all the sequence information responsible for the Er-MAPK1 subcellular localization. However, in fibroblasts and S. pombe this information determined a nucleolar localization of the GFP-tagged C-terminal domain, and a ciliary localization in T. thermophila. In the light of these findings, the Er-MAPK1 localization in E. raikovi was re-examined via immunoreactions and shown to be ciliary besides that nuclear, as is the case for the mammalian intestinal cell kinase with which the Er-MAPK1 N-terminal domain shares a strong sequence identity and a catalytic function.

Keywords: ciliated protozoa, protein kinase, heterologous gene expression, GFP-fusion proteins, nuclear and ciliary protein kinase, ICK-related kinase, MAK-related kinase

\section{INTRODUCTION}

Protist ciliates possess remarkably high numbers of genes encoding protein kinases. The genomes of Tetrahymena thermophila, Paramecium tetraurelia, and Stentor coeruleus contain 1069, 2606, and 2057 kinase-coding genes, respectively, representing 4, 6.6, and 6\% of the whole gene number (Eisen et al., 2006; Bemm et al., 2009; Reiff and Marshall, 2017). In man, in contrast, the 518 kinase-coding genes approximate to only $1.7 \%$ of all genes (Manning et al., 2002). However, which subcellular localization and function distinguish the products encoded by this multitude of ciliate kinase-coding genes are practically unexplored questions.

In the transcriptionally active somatic nucleus (macronucleus) of a free-living marine ciliate, Euplotes raikovi, a 631-amino acid protein kinase, designated Er-MAPK1, was identified in functional relation with the cell switching between the asexual and sexual stages of the life cycle, it appearing phosphorylated in vegetative cells growing stimulated by autocrine (autologous) interactions with their secreted (self) water-borne signaling protein pheromone and 
de-phosphorylated in cells temporarily induced to unite in mating pairs by competitive paracrine (heterologous) interactions with a non-self pheromone (Vallesi et al., 2010).

The site of the Er-MAPK1 enzymatic activity was clearly identified with the 283-amino acid N-terminal domain. Its sequence carries all the sub-domains that are common to serine/threonine protein kinases and, located in the activation loop, the Thr-Xxx-Tyr motif which is distinctive of the Mitogen-Activated Protein Kinases (MAPKs) widely conserved in eukaryotic cells and involved in regulating an ample variety of cellular processes including proliferation, differentiation, motility, stress response, and apoptosis (Plotnikov et al., 2011).

Differently from the Er-MAPK1 N-terminal domain, the 348-amino acid C-terminal domain appeared to be structurally unique and this uniqueness raised the question of which functions it plays. A function in driving the Er-MAPK1 nuclear localization was inferred by observing its extremely basic amino acid composition (isoelectric point, 10.2).

Considering a persistent lack of a reliable procedure to introduce, maintain, and faithfully express a modified chromosome into Euplotes cells, the above hypothesis was assessed by linking the Green-Fluorescent-Protein (GFP) to the Er-MAPK1 N- and C-terminal domains, and expressing the two tagged domains in three distinct heterologous systems represented by mammalian fibroblasts, the yeast Schizosaccharomyces pombe and the ciliate Tetrahymena thermophila. In addition to obtaining convergent evidence that the information for the Er-MAPK1 subcellular localization resides exclusively in the protein C-terminal domain, the expression in Tetrahymena revealed that this information may determine a ciliary beside that a nuclear localization.

\section{MATERIALS AND METHODS}

\section{Analysis of Protein Structure and Prediction of Nuclear Localization Signals (NLSs)}

Protein sequence analysis and NLS prediction were performed in silico, using the internet website interfaces provided by the following prediction programs: InterPro: http: //www.ebi.ac.uk/interpro/ (Mitchell et al., 2019); DICHOT: http: //www.ideal.force.cs.is.nagoya-u.ac.jp/dichot/ (Fukuchi et al., 2011); PSORT II: https://psort.hgc.jp/form2.html (Nakai and Horton, 1999); Eukaryotic Linear Motif resources (ELM): http://elm.eu.org/search.html (Gouw et al., 2018); NLSmapper: http://nls-mapper.iab.keio.ac.jp/cgi-bin/NLS_Mapper_form.cgi (Kosugi et al., 2009).

\section{Amplification and Construction of Expression Vectors}

The full-length Er-MAPK1 sequence and segments encoding the Er-MAPK1 N- and C-terminal domains were amplified from the Er-MAPK1 gene cloned into the pCR2.1-TOPO vector (Invitrogen) as previously described (Vallesi et al., 2010). No TGA stop codon (specifying cysteine in Euplotes) is present in the Er-MAPK1 gene coding region, and the +1 -frameshifting site was removed by single-base deletion. Primers used in PCR amplifications (Table 1) were designed in such a way to add a restriction site to the $5^{\prime}$ end. Fragments amplified with primers containing $\mathrm{BamHI}$ and $\mathrm{Xba \textrm {I }}$ restriction sites were cloned into the pVAX1 vector (Invitrogen), provided with both a human cytomegalovirus immediate-early promoter for a high-level protein expression and a bovine growth hormone polyadenylation signal for an efficient mRNA transcription termination. Fragments amplified with primers with SmaI restriction site into the thiamine-repressible pREP41 vector (Maundrell, 1993), and fragments amplified with primers with XhoI and ApaI restriction sites into the ribosomal DNAbased vector PIGF1 under the cadmium-inducible MTT1 gene promoter (Shang et al., 2002; Malone et al., 2005). The correctness of constructs in each generated expression vector was confirmed by DNA sequencing.

\section{Transfection of Mammalian Fibroblasts}

Fibroblasts of the NIH3T3 cell line were cultured in Dulbecco's modified Eagle's medium (DMEM) with GlutaMax-I supplemented with $10 \%$ fetal bovine serum (Invitrogen), $100 \mathrm{U} / \mathrm{ml}$ penicillin and $100 \mu \mathrm{g} / \mathrm{ml}$ streptomycin, and split every 2-4 days to maintain monolayer coverage. Twenty-four hours before transfection, 250,000 cells were seeded onto a sterile glass coverslips in 12-well culture plates, using medium without antibiotics and incubated in order to reach $70-80 \%$ confluence. Lipoplexes used for transfection were prepared in Opti-MEM I Reduced Serum Medium (Invitrogen) by mixing $2 \mu \mathrm{g}$ of plasmid with $4 \mu \mathrm{l}$ of Lipofectamine 2000 Reagent (Invitrogen) for each well. Cells were incubated in Opti-MEM for $4 \mathrm{~h}$, then washed with phosphate buffer saline (PBS: $130 \mathrm{mM}$ $\mathrm{NaCl}, 2 \mathrm{mM} \mathrm{KCl}, 8 \mathrm{mM} \mathrm{Na}_{2} \mathrm{HPO}_{4}, 2 \mathrm{mM} \mathrm{KH}_{2} \mathrm{PO}_{4}, \mathrm{pH}$ 7.2) before being incubated in growth medium for $48 \mathrm{~h}$. Transfected fibroblasts were fixed with $4 \%$ paraformaldehyde in PBS for $10 \mathrm{~min}$ at room temperature, and incubated with DAPI (Sigma-Aldrich) to visualize the nuclei. Coverslips were then mounted with Mowiol 4-88 (Calbiochem) onto microscope slides and observed on Nikon inverted microscope (DiaphotTMD) connected to a laser-scanning confocal imaging system (MRC 600, Bio-Rad).

\section{Transfection of S. pombe}

Schizosaccharomyces pombe cells of strain TGO509 ( $h^{90}$ leu1 gar2-mCherry:kanR) were transfected as previously described (Moreno et al., 1991). This strain is auxotrophic for leucine,

TABLE 1 | Primers used in this study.

\begin{tabular}{lll}
\hline Gene sequence & Forward primer $\left(\mathbf{5}^{\prime} \mathbf{-} \mathbf{3}^{\prime}\right)$ & Reverse primer $\left(\mathbf{5}^{\prime} \mathbf{-} \mathbf{3}^{\prime}\right)$ \\
\hline GFP & ATGGCTAGCAAAG & GTTGTACAGTTC \\
& GAGAAGAACTC & ATCCATGCC \\
N-terminal domain & ATGGACAGGTACA & TAAGAGTAGTCA \\
& AGATAATC & TGCTCCAGCAA \\
C-terminal domain & TTAAATITGACC & AAAGATITGTG \\
& CATATCT & CCGCCAGTC
\end{tabular}


and transfection with pREP41-based GFP-Er-MAPK1 expression vectors can complement this leucine auxotrophy. Therefore, transfected cells were selected on solid minimal medium (EMM2) lacking leucine and supplemented with $2 \mu \mathrm{M}$ thiamine to repress GFP-Er-MAPK1 constructs expression, and cultured in EMM2 without thiamine to induce GFP-Er-MAPK1 constructs expression. Living cells were then pressed between two coverslips and observed using a fluorescence microscope IX-70 equipping a PlanApo/60x/NA1.4 oil SC objective (Olympus, Tokyo, Japan). Fluorescence images were obtained by the DeltaVision system (Applied Precision, Issaquah, WA, United States).

\section{Transfection of T. thermophila}

Conjugating pairs between cell strains CU427 [chx1-1/chx1-1 (CHX1; cy-s, VI)] and CU428 [mpr1-1/mpr1-1 (MPR1; mp$\mathrm{s}, \mathrm{VII}$ )] were transfected as previously described (Iwamoto et al., 2014). Transfected cells were diluted into culture medium composed of $1.5 \%(\mathrm{w} / \mathrm{v})$ proteose-peptone, $0.5 \%(\mathrm{w} / \mathrm{v})$ yeast extract, and $0.5 \%(\mathrm{w} / \mathrm{v}) \mathrm{D}$-glucose (Difco) and aliquoted to 96 wells plates. After incubation at $30^{\circ} \mathrm{C}$ for $18 \mathrm{~h}$, paromomycin sulfate (Duchefa Biochemie) was added as a selective drug at the final concentration of $120 \mu \mathrm{g} / \mathrm{ml}$. Successfully transformed cells exhibiting paromomycin resistance grew after 3 days of selective cultivation. $\mathrm{CdCl}_{2}$ at concentrations of $1.0-2.0 \mu \mathrm{g} / \mathrm{ml}$ was added to the culture in logarithmic growth phase at approximately $3 \mathrm{~h}$ before observation. Living cells were observed using a fluorescence microscope IX-70 equipping a UApo/40x/NA1.35 objective (Olympus, Tokyo, Japan).

\section{Immunofluorescence Analysis}

Immunofluorescence analysis of transfected fibroblasts was performed to visualize nucleoli, using anti-fibrillarin antibodies (Thermo-Fisher). Briefly, cells were fixed with 4\% paraformaldehyde, permeabilized with $0.2 \%$ Triton-X-100 in PBS for $5 \mathrm{~min}$, blocked for $30 \mathrm{~min}$ in 1\% BSA in PBS, and incubated for $1 \mathrm{~h}$ with the primary antibody, followed by incubation for $1 \mathrm{~h}$ with the DyLight 594-conjugated anti-mouse antibodies (Bethyl Laboratories, Inc.). Samples were washed with PBS, then mounted with Mowiol 4-88 (Calbiochem) onto microscope slides for observation.

To analyze Er-MAPK1 ciliary localization, E. raikovi cells were incubated with Triton-X in microtubule-stabilizing buffer (60 mM PIPES, $25 \mathrm{mM}$ HEPES, $10 \mathrm{mM}$ EGTA, $2 \mathrm{mM} \mathrm{MgCl}$, pH 6.9), as previously described (Kloetzel et al., 2003). Cells were then fixed on slides with $80 \%$ ethanol, rehydrated in PBS and incubated at room temperature for $1 \mathrm{~h}$ with $1 \%$ bovine serum albumin (BSA) and a second hour with a customsynthesized antibodies (anti- $\mathrm{S}_{489}-\mathrm{R}_{502}$ ) (GenScript) directed against the sequence Ser 489 -Arg-Lys-Asp-Ser-Arg-Lys-Glu-SerArg-Lys-Asp-Ser-Arg 502 which is exclusive of the Er-MAPK1 C-terminal domain. After washing with PBS, samples were finally incubated for $1 \mathrm{~h}$ with DyLight 488-conjugated antirabbit antibodies (Bethyl Laboratories, Inc.) washed again, and mounted onto microscope slides for observation.

\section{RESULTS}

\section{Er-MAPK1 Structure and Putative NLSs}

Amino acid sequence and structural motifs of Er-MAPK1 are shown in Figure 1. Two different protein domains are clearly identified by in silico analyses. The $\mathrm{Met}_{1}$ to $\mathrm{Phe}_{283} \mathrm{~N}$-terminal domain contains the eleven sub-domains for the kinase catalytic activity. The $\mathrm{Lys}_{284}$ to $\mathrm{Phe}_{631} \mathrm{C}$-terminal domain is predicted

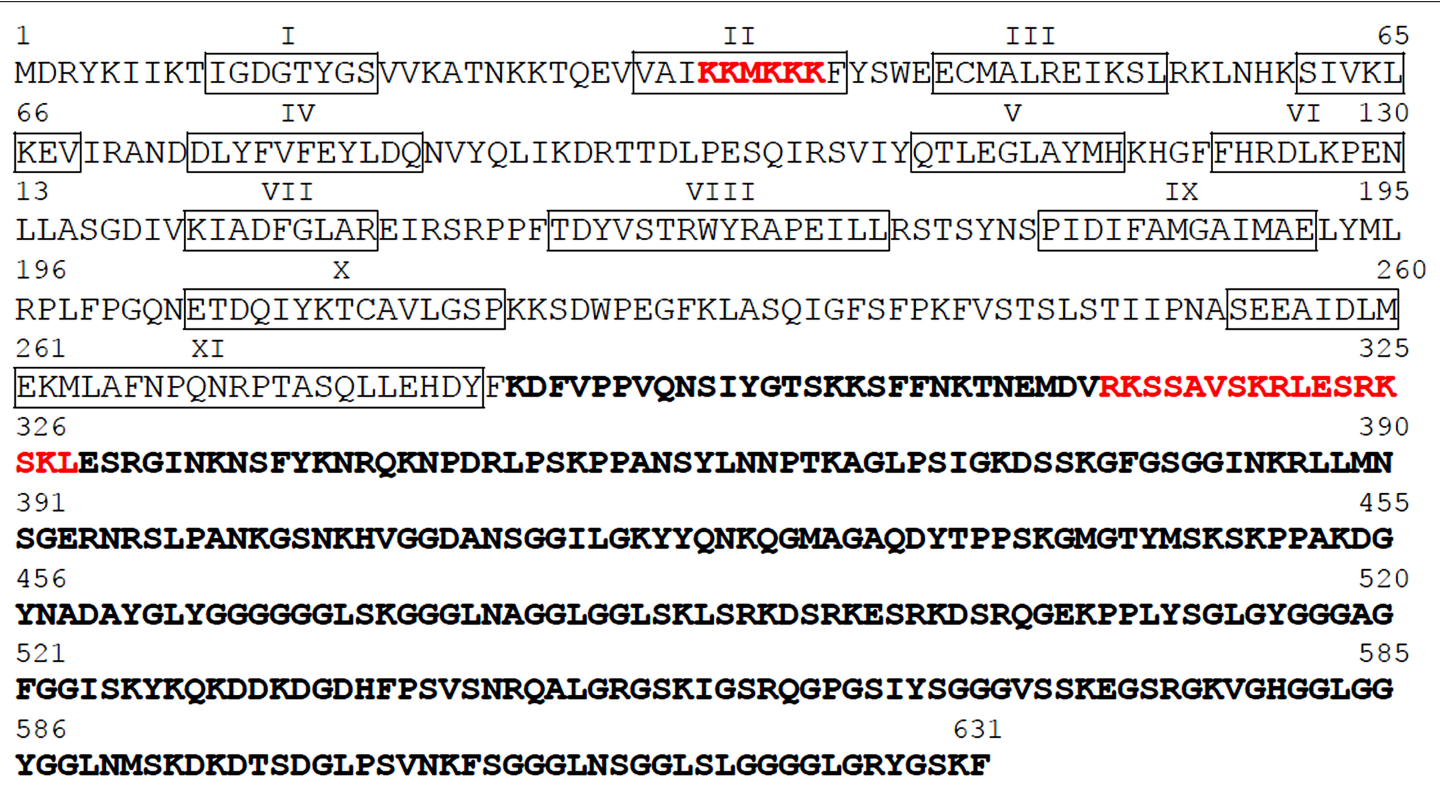

FIGURE 1 | Amino acid sequence of Er-MAPK1. The N- and C-terminal domains are written with regular and bold letters, respectively. The eleven MAPK-specific sub-domains are boxed and indicated by roman numbers; predicted NLS sequences by the programs PSORT II (score, 0.51), ELM motif search (probability, 2.588 $\mathrm{e}^{-04}$ ), and NLSmapper (score 4.7) are written in red. 


\title{
A Fibroblasts
}
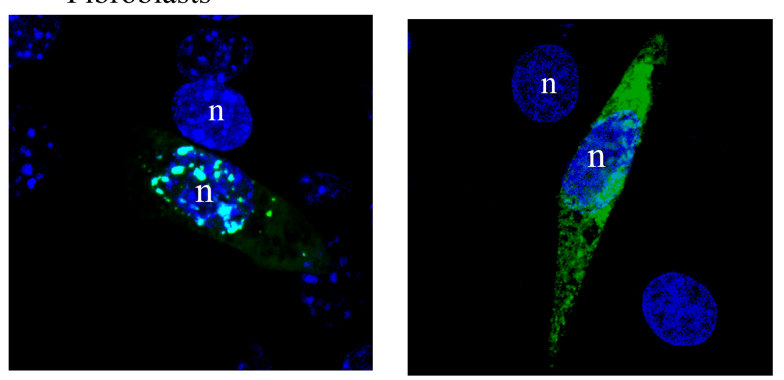

GFP-Er-MAPK1 (full length) + DAPI
GFP-Er-MAPK1 N-terminal + DAPI

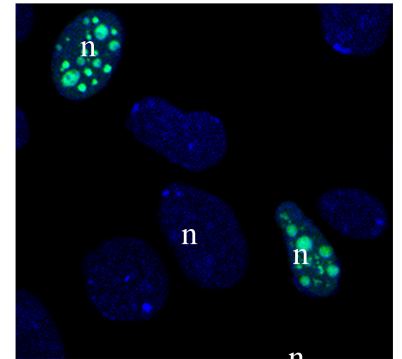

GFP-Er-MAPK1 C-terminal + DAPI

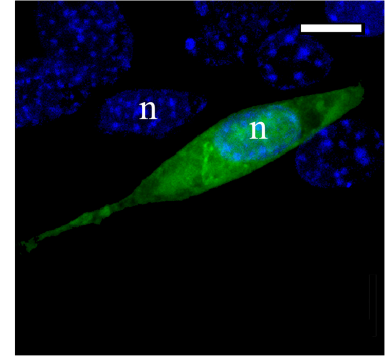

GFP + DAPI

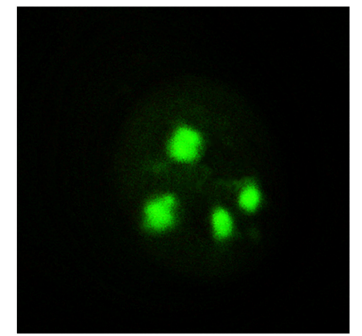

GFP-Er-MAPK1 C-terminal

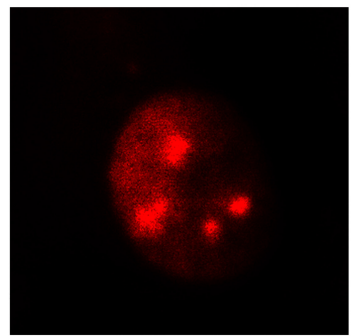

anti-fibrillarin

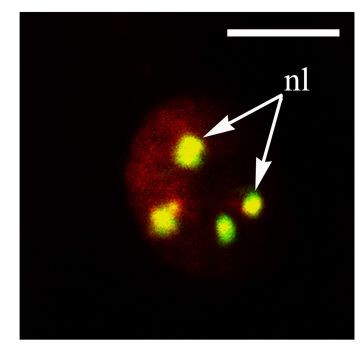

merge

B

\begin{abstract}
Schizosaccharomyces pombe
\end{abstract}

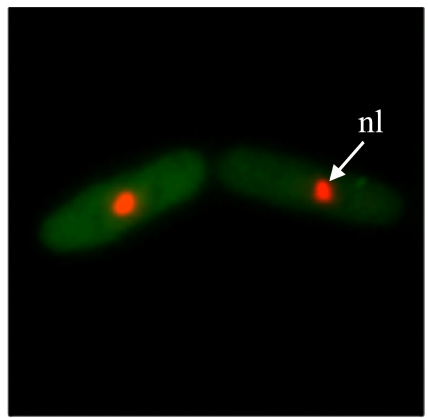

GFP-Er-MAPK1 N-terminal + Gar2 mCherry

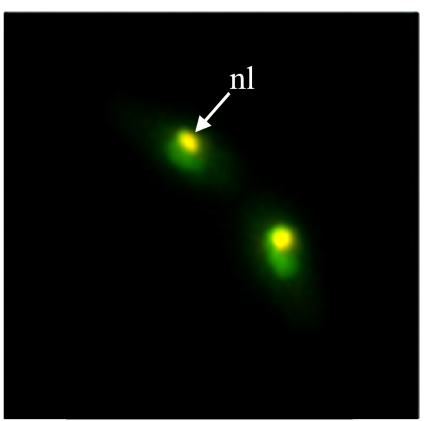

GFP-Er-MAPK1 C-terminal + Gar2 mCherry

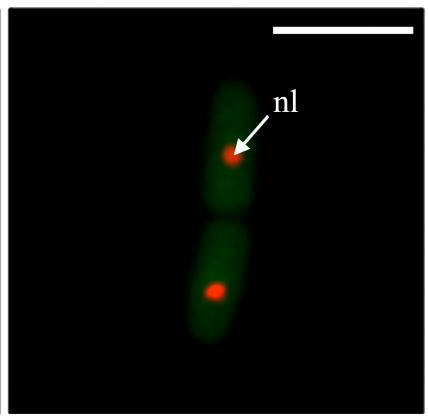

GFP + Gar2 mCherry

c Tetrahymena thermophila

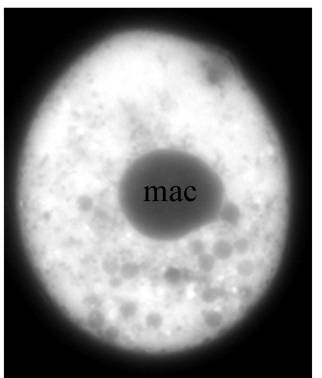

GFP-Er-MAPK1 N-terminal

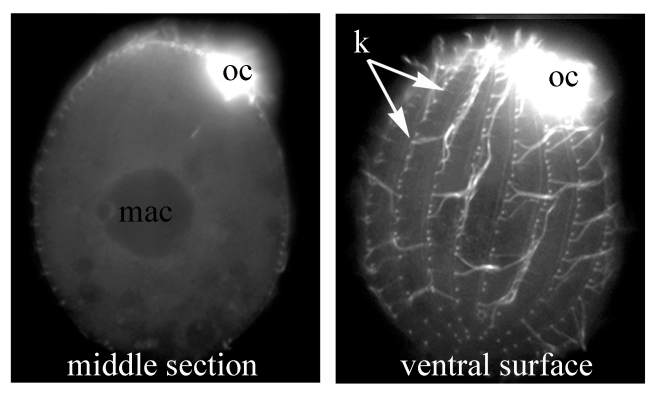

GFP-Er-MAPK1 C-terminal

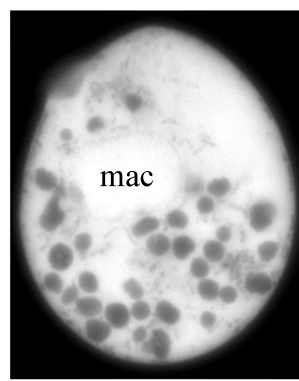

GFP

FIGURE 2 | Heterologous expression of GFP-tagged Er-MAPK1 domains. (A) Top: representative fluorescence images of transfected fibroblasts (green). Nuclei are stained with DAPI (blue). GFP-Er-MAPK1 full-length and GFP-C-terminal proteins localize into the nucleus, and the GFP-N-terminal protein in the cytoplasm. GFP alone, used as control, diffuses in both cytoplasm and nucleus due to its molecular weight ( $28 \mathrm{kDa}$ ) below the exclusion size of nuclear pores. Bottom: magnification of a fibroblast nucleus in which the GFP-C-terminal protein (green) and anti-fibrillarin antibodies (red) co-localize in nucleoli (yellow). Each image represents a single section. (B) Representative fluorescence images of S. pombe cells co-expressing the GFP-N-terminal protein, the GFP-C-terminal protein and GFP alone (green) with the nucleolar protein Gar2 (red). The nucleolar co-localization (central picture) between the GFP-C-terminal protein and the Gar2 protein is yellow. (C) Representative fluorescence images of $T$. thermophila expressing the GFP-N-terminal protein, the GFP-C-terminal protein and GFP alone. The GFP-C-terminal protein accumulates in the oral ciliature and single somatic cilia. n, nucleus; nl, nucleolus; mac, macronucleus; oc, oral ciliature; k, kinetosomes in a ciliary row; bars, $10 \mu \mathrm{m}$. 
to have an intrinsically disordered configuration, a structural feature which is considered suitable for the interaction with other proteins by exposing short linear peptide motifs (Babu et al., 2012; van der Lee et al., 2014). Commonly used webbased prediction programs recognized two distinct NLS motifs. One lies in the protein N-terminal domain and is represented by the hexapeptide Lys33-Lys-Met-Lys-Lys-Lys 38 matching the general consensus sequence Lys(Lys/Arg) Xxx(Lys/Arg) of monopartite NLS motifs (Lange et al., 2007). It is internal to subdomain II responsible for the phosphor-transfer activity of the kinase. A second predicted motif, Arg 312 -Lys-Ser-Ser-AlaVal-Ser-Lys-Arg-Leu-Glu-Ser-Arg-Lys-Ser-Lys-Leu 328 , resides in the Er-MAPK1 C-terminal region and is recognized as a bipartite variant of the classical basically charged NLS (Dingwall and Laskey, 1991; Robbins et al., 1991). It is formed by two basic residues a 10-residue spacer, and another basic region consisting of three basic residues out of five residues.

\section{Expression in Fibroblasts}

In murine fibroblasts of the NIH3T3 line, the individual expression of the Er-MAPK1 N-terminal and C-terminal domains was carried out in parallel with the expression of the fulllength $E r$-MAPK1 sequence in such a way to preliminarily verify the capability of a heterologous system to accomplish a correct Er-MAPK1 nuclear localization. The transfection was run by ligating the coding sequences of the three proteins downstream and in-frame with the GFP-coding gene, and inserting the ligated sequences into the mammalian expression vector pVAX. Analyzed in confocal microscopy (Figure 2A), transfected fibroblasts revealed an equal spotted nuclear localization for both the entire Er-MAPK1 protein and the C-terminal domain, and the spots were shown to be nucleoli by proving co-localization between the GFP signal of the fusion proteins and the immunestaining generated by fibrillarin antibodies used as nucleolar marker. In contrast, the N-terminal fusion protein appeared uniformly dispersed throughout the cell cytoplasm, implying that no specific sequence motif of the Er-MAPK1 N-terminal domain, and the predicted NLS in particular, are involved in the protein nuclear localization.

\section{Expression in S. pombe}

The expression of the GFP-Er-MAPK1 constructs in S. pombe was performed in cells synthesizing the nucleolar Gar2 protein tagged with the red fluorescent protein mCherry (Gulli et al., 1995), using construct copies amplified via PCR from the vector pVAX1 and ligated into the multi-cloning site of vector pREP41 (Maundrell, 1993). Transfected S. pombe cells were analyzed in fluorescence microscopy after having been cultivated for $15 \mathrm{~h}$ in absence of thiamine to induce protein synthesis. Fully matching the transfected fibroblasts, they showed (Figure 2B) the GFP-Cterminal protein concentrated inside the nucleus and, in contrast, the GFP-N-terminal protein uniformly dispersed throughout the cell cytoplasm. The GFP-C-terminal protein association to nucleoli was in this case validated by showing co-localization between the GFP signal and the mCherry fluorescence of the Gar2 protein.

\section{Expression in T. thermophila and Er-MAPK1 Immunolocalization in E. raikovi}

For the expression in T. thermophila, the GFP-Er-MAPK1 constructs were inserted into the vector-pIGF1, and cells were

\section{Euplotes raikovi}

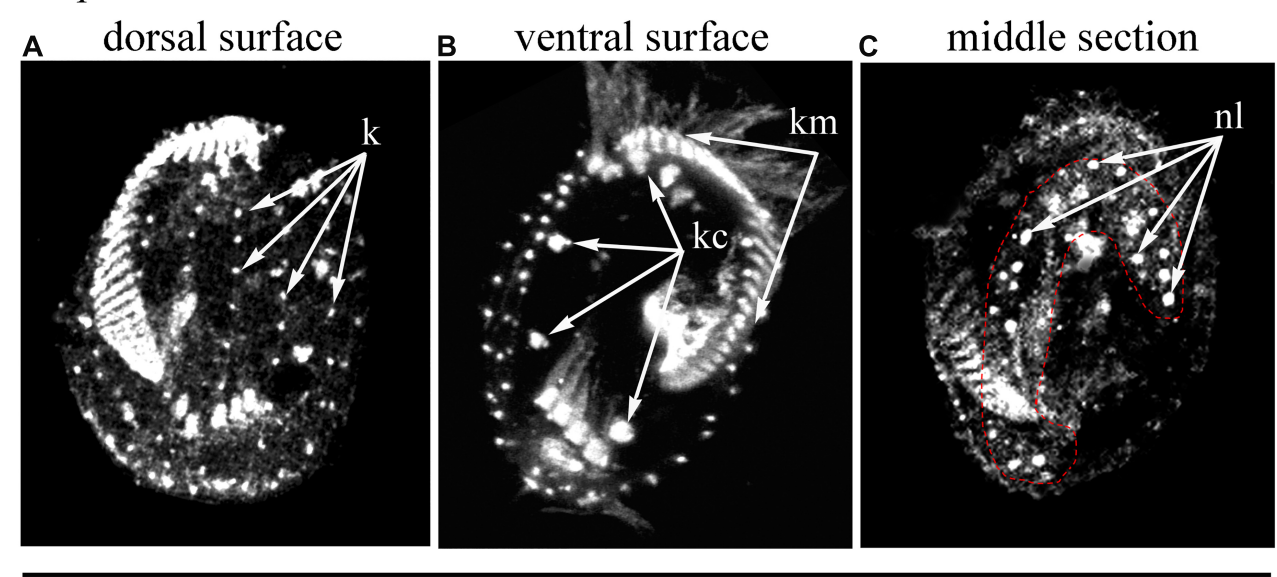

anti-S489-R502 Abs

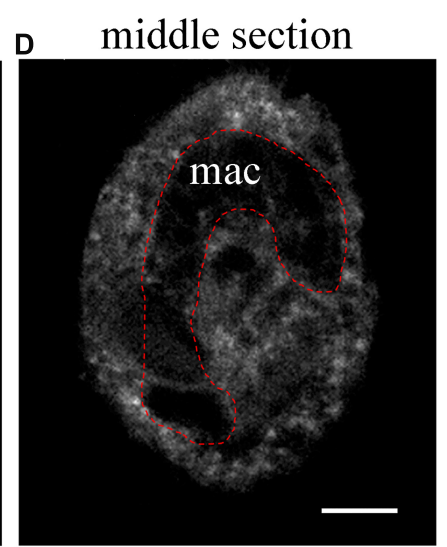

anti-S489-R502 Abs $+\mathrm{S}_{489}-\mathrm{R}_{502}$ peptide

FIGURE 3 | Localization of Er-MAPK1 in E. raikovi. Representative images of cells incubated with antibodies directed against the sequence Ser 489 -Arg502 of the C-terminal domain (A-C), and a mixture between the same antibodies and the Ser 489 -Arg 502 peptide (D). The position of the macronucleus is outlined by a red dotted line. k, kinetosomes of dorsal cilia (bristles); kc and km, packed kinetosomes of cirri and membranelles, respectively; nl, nucleolus; mac, macronucleus; bar, $10 \mu \mathrm{m}$. 
analyzed under fluorescence microscope 3 days after transfection and exposure to $\mathrm{CdCl}_{2}$. Alike fibroblasts and $S$. pombe, transfected $T$. thermophila cells revealed a specific subcellular localization only of the GFP-C-terminal protein. However, this localization was ciliary, not nucleolar (Figure 2C). The fluorescent signal revealed a particularly intense association to the kinetosomes of the somatic single cilia covering the cell body surface, and produced a massive (unresolved) staining at level of the oral membranelles surrounding numerous the cell cytostome.

In the light of this ciliary localization of the GFP-Cterminal protein in $T$. thermophila, the native localization of the entire Er-MAPK1 protein was re-examined in E. raikovi via immunoreactions carried out with antibodies generated against a strong immunogenic, Er-MAPK1-specific sequence,

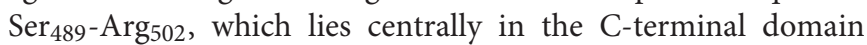
and finds no significant counterpart in other proteins. As shown in Figure 3, in addition to decorating nucleoli in the cell macronucleus, these antibodies bound intensely also to the kinetosomes of the whole (somatic and oral) E. raikovi ciliature, represented by the single bristle cilia on the cell dorsal surface and compound ciliary organelles (cirri and adoral membranelles) on the ventral surface. From these immunoreactions it was thus evident that the Er-MAPK1 subcellular localization in E. raikovi is not exclusively nuclear, but also ciliary.

\section{DISCUSSION}

By heterologous expression of GFP-tagged N- and C-terminal proteins in mammalian fibroblasts, S. pombe and T. thermophila, we provided evidence that (i) the subcellular localization of the E. raikovi Er-MAPK1 protein kinase depends on sequence information residing exclusively in the structurally unique, extremely basic C-terminal domain, and (ii) this domain may target the protein to the nucleus in specific association with nucleoli as originally reported in the native organism, as well as to cilia in apparently preferential association with the kinetosomes.

To transit into the nucleus and cilia, proteins rely on NLS sequences which are recognized and bound by importins that govern their passage through nuclear pore complexes and ciliary gates (Dishinger et al., 2010; Kee et al., 2012; Kee and Verhey, 2013; Bauer et al., 2015; Del Viso et al., 2016). The $\operatorname{Arg}_{312}-\operatorname{Leu}_{328}$ sequence identified in the ErMAPK1 C-terminal domain as NLS by predictive programs presents all the requisites to act as key site of interactions with importins. It contains two clusters of basic amino acids separated by a short spacer region as classical bipartite NLSs, and is well exposed on the molecular surface, hence capable to interact with transporter proteins. In addition, it appears to be closely conserved among Er-MAPK1 homologs of other Euplotes species such as E. vannus (Chen et al., 2019), E. nobilii (Candelori et al., 2013) and E. petzi (unpublished results), as well as among Er-MAPK1 analogs characterized from other ciliates. The conclusion that the $\operatorname{Arg}_{312}-$Leu$_{328}$ sequence is directly responsible for the $\mathrm{Er}$ MAPK1 subcellular localization, is not necessarily weakened by the observation that $T$. thermophila localized the $E r$ MAPK1 GFP-C-terminal protein to cilia, and not to the nucleus. T. thermophila is known to have a peculiar mechanism of nuclear entry, with several "universal" NLSs that are not recognized by transporter proteins and Tetrahymena NLSs that have no function in other eukaryotic cells (Iwamoto et al., 2018).

Among serine/threonine kinases more closely comparable to Er-MAPK1 in amino acid sequence, more significant relationships were found to link Er-MAPK1 to the flagellar kinase MAPK7 identified from the Chlamydomonas genome (Merchant et al., 2007), and the mammalian Male-germ cell Associated Kinases (MAKs) and Intestinal Cell Kinases (ICKs), initially identified in relation to mechanisms underlying the proliferative and developmental cell response to growth factors (Togawa et al., 2000; Ma et al., 2006; Fu et al., 2009), and later detected also in cilia where they act as negative regulators of the ciliary length and ciliogenesis (Chaya et al., 2014; Moon et al., 2014; Oud et al., 2016). In a functional perspective, however, the Er-MAPK1 relationships with mammalian ICKs and MAKs appeared to be much more meaningful than the relationships with MAPK7, considering that this kinase has an extra-nuclear localization and an amino acid sequence that, lacking a distinct C-terminal domain, extends only half of the ErMAPK1 sequence. This conclusion is here reinforced by the experimental evidence that Er-MAPK1, in addition to showing the same double phosphorylation Thr-AspTyr site as ICKs and MAKs and a matching sequence length and organization, shares also a double, nuclear and ciliary, subcellular localization, which suggests to credit this Euplotes kinase with an early evolved multiple, contextdependent activity.

\section{DATA AVAILABILITY STATEMENT}

All datasets generated for this study are included in the manuscript/supplementary files.

\section{AUTHOR CONTRIBUTIONS}

AC, AA, and AV conceived and planned the study. AC, TY, MI, and $\mathrm{MM}$ performed the experiments and analyzed the data. AC, TY, and MI prepared the figures. AV wrote the manuscript with the contribution of all authors.

\section{ACKNOWLEDGMENTS}

We gratefully acknowledge Prof. Pierangelo Luporini for critical discussions and helpful suggestions and Dr. Patrizia Ballarini for confocal microscopy analysis assistance. 


\section{REFERENCES}

Babu, M. M., Kriwacki, R. W., and Pappu, R. V. (2012). Versatility from protein disorder. Science 337, 1460-1461. doi: 10.1126/science.122 8775

Bauer, N. C., Doetsch, P. W., and Corbett, A. H. (2015). Mechanisms regulating protein localization. Traffic 16, 1039-1061. doi: 10.1111/tra. 12310

Bemm, F., Schwarz, R., Forster, F., and Schultz, J. (2009). A kinome of 2600 in the ciliate Paramecium tetraurelia. FEBS Lett. 583, 3589-3592. doi: 10.1016/j.febslet. 2009.10.029

Candelori, A., Luporini, P., Alimenti, C., and Vallesi, A. (2013). Characterization and expression of the gene encoding En-MAPK1, an intestinal cell kinase (ICK)-like kinase activated by the autocrine pheromone-signaling loop in the polar ciliate, Euplotes nobilii. Int. J. Mol. Sci. 14, 7457-7467. doi: 10.3390/ ijms 14047457

Chaya, T., Omori, Y., Kuwahara, R., and Furukawa, T. (2014). ICK is essential for cell type-specific ciliogenesis and the regulation of ciliary transport. EMBO J. 33, 1227-1242. doi: 10.1002/embj.201488175

Chen, X., Jiang, Y., Gao, F., Zheng, W., Krock, T. J., Stover, N. A., et al. (2019). Genome analyses of the new model protist Euplotes vannus focusing on genome rearrangement and resistance to environmental stressors. Mol. Ecol. Resour. 19, 1292-1308. doi: 10.1111/1755-0998.13023

Del Viso, F., Huang, F., Myers, J., Chalfant, M., Zhang, Y., Reza, N., et al. (2016). Congenital heart disease genetics uncovers context-dependent organization and function of nucleoporins at cilia. Dev. Cell 38, 478-492. doi: 10.1016/j.devcel. 2016.08.002

Dingwall, C., and Laskey, R. A. (1991). Nuclear targeting sequences-a consensus? Trends Biochem. Sci. 16, 478-481. doi: 10.1016/0968-0004(91)90 184-W

Dishinger, J. F., Kee, H. L., Jenkins, P. M., Fan, S., Hurd, T. W., Hammond, J. W., et al. (2010). Ciliary entry of the kinesin-2 motor KIF17 is regulated by importin-beta2 and RanGTP. Nat. Cell Biol. 12, 703-710. doi: 10.1038/ncb 2073

Eisen, J. A., Coyne, R. S., Wu, M., Wu, D., Thiagarajan, M., Wortman, J. R., et al. (2006). Macronuclear genome sequence of the ciliate Tetrahymena thermophila, a model eukaryote. PLoS Biol. 4:286. doi: 10.1371/journal.pbio.004 0286

Fu, Z., Kim, J., Vidrich, A., Sturgill, T. W., and Cohn, S. M. (2009). Intestinal cell kinase, a MAP kinase-related kinase, regulates proliferation and G1 cell cycle progression of intestinal epithelial cells. Am. J. Physiol. Gastrointest. Liver Physiol. 297, G632-G640. doi: 10.1152/ajpgi.00066. 2009

Fukuchi, S., Hosoda, K., Homma, K., Gojobori, T., and Nishikawa, K. (2011). Binary classification of protein molecules into intrinsically disordered and ordered segments. BMC Struct. Biol. 11:29. doi: 10.1186/1472-680711-29

Gouw, M., Michael, S., Sámano-Sánchez, H., Kumar, M., Zeke, A., Lang, B., et al. (2018). The eukaryotic linear motif resource-2018 update. Nucleic Acids Res. 46, D428-D434. doi: 10.1093/nar/gkx1077

Gulli, M. P., Girard, J. P., Zabetakis, D., Lapeyre, B., Melese, T., and Caizergues-Ferrer, M. (1995). Gar2 is a nucleolar protein from Schizosaccharomyces pombe required for $18 \mathrm{~S}$ rRNA and $40 \mathrm{~S}$ ribosomal subunit accumulation. Nucleic Acids Res. 23, 1912-1918. doi: 10.1093/nar/23.11. 1912

Iwamoto, M., Mori, C., Hiraoka, Y., and Haraguchi, T. (2014). Puromycin resistance gene as an effective selection marker for ciliate Tetrahymena. Gene 534, 249-255. doi: 10.1016/j.gene.2013.10.049

Iwamoto, M., Mori, C., Osakada, H., Koujin, T., Hiraoka, Y., and Haraguchi, T. (2018). Nuclear localization signal targeting to macronucleus and micronucleus in binucleated ciliate Tetrahymena thermophila. Genes Cells 23, 568-579. doi: 10.1111 /gtc. 12602

Kee, H. L., Dishinger, J. F., Blasius, T. L., Liu, C. J., Margolis, B., and Verhey, K. J. (2012). A size-exclusion permeability barrier and nucleoporins characterize a ciliary pore complex that regulates transport into cilia. Nat. Cell Biol. 14, 431-437. doi: $10.1038 /$ ncb2450

Kee, H. L., and Verhey, K. J. (2013). Molecular connections between nuclear and ciliary import processes. Cilia 2:11. doi: 10.1186/2046-2530-2-11
Kloetzel, J. A., Baroin-Tourancheau, A., Miceli, C., Barchetta, S., Farmar, J., Banerjee, D., et al. (2003). Cytoskeletal proteins with N-terminal signal peptides: plateins in the ciliate Euplotes define a new family of articulins. J. Cell Sci. 116, 1291-1303. doi: 10.1242/jcs.00333

Kosugi, S., Hasebe, M., Tomita, M., and Yanagawa, H. (2009). Systematic identification of yeast cell cycle-dependent nucleocytoplasmic shuttling proteins by prediction of composite motifs. Proc. Natl. Acad. Sci. U.S.A. 106, 10171-10176. doi: 10.1073/pnas.090060 4106

Lange, A., Mills, R. E., Lange, C. J., Stewart, M., Devine, S. E., and Corvett, A. H. (2007). Classical nuclear localization signals: definition, function, and interaction with importin alpha. J. Biol. Chem. 282, 5101-5105. doi: 10.1074/ jbc.R600026200

Ma, A. H., Xia, L., Desai, S. J., Boucher, D. L., Guan, Y., Shih, H. M., et al. (2006). Male germ cell-associated kinase, a male- specific kinase regulated by androgen, is a coactivator of androgen receptor in prostate cancer cells. Cancer Res. 66, 8439-8447.

Malone, C. D., Anderson, A. M., Motl, J. A., Rexer, C. H., and Chalker, D. L. (2005). Germ line transcripts are processed by a Dicer-like protein that is essential for developmentally programmed genome rearrangements of Tetrahymena thermophila. Mol. Cell. Biol. 25, 9151-9164. doi: 10.1128/MCB.25.20.91519164.2005

Manning, G., Whyte, D. B., Martinez, R., Hunter, T., and Sudarsanam, S. (2002). The protein kinase complement of the human genome. Science 298, 1912-1934. doi: $10.1126 /$ science. 1075762

Maundrell, K. (1993). Thiamine-repressible expression vectors pREP and pRIP for fission yeast. Gene 123, 127-130. doi: 10.1016/0378-1119(93)90 551-D

Merchant, S. S., Prochnik, S. E., Vallon, O., Harris, E. H., Karpowicz, S. J., Witman, G. B., et al. (2007). The Chlamydomonas genome reveals the evolution of key animal and plant functions. Science 318, 245-250. doi: 10.1126/science.114 3609

Mitchell, A. L., Attwood, T. K., Babbitt, P. C., Blum, M., Bork, P., Bridge, A., et al. (2019). InterPro in 2019: improving coverage, classification and access to protein sequence annotations. Nucleic Acids Res. 8, D351-D360. doi: 10.1093/ nar/gky1100

Moon, H., Song, J., Shin, J. O., Lee, H., Kim, H. K., Eggenschwiller, J. T., et al. (2014). Intestinal cell kinase, a protein associated with endocrine-cerebroosteodysplasia syndrome, is a key regulator of cilia length and Hedgehog signaling. Proc. Natl. Acad. Sci. U.S.A. 111, 8541-8546. doi: 10.1073/pnas. 1323161111

Moreno, S., Klar, A., and Nurse, P. (1991). Molecular genetic analysis of fission yeast Schizosaccharomyces pombe. Methods Enzymol. 194, 795-823. doi: 10. 1016/0076-6879(91)94059-L

Nakai, K., and Horton, P. (1999). PSORT: a program for detecting the sorting signals of proteins and predicting their subcellular localization. Trends Biochem. Sci. 24, 34-35. doi: 10.1016/s0968-0004(98)01 336-x

Oud, M. M., Bonnard, C., Mans, D. A., Altunoglu, U., Tohari, S., Ng, A. Y. J., et al. (2016). A novel ICK mutation causes ciliary disruption and lethal endocrinecerebro-osteodysplasia syndrome. Cilia 5:8. doi: 10.1186/s13630-0160029-1

Plotnikov, A., Zehorai, E., Procaccia, S., and Seger, R. (2011). The MAPK cascades: signaling components, nuclear roles and mechanisms of nuclear translocation. Biochim. Biophys. Acta 1813, 1619-1633. doi: 10.1016/j.bbamcr.2010.12.012

Reiff, S. B., and Marshall, W. F. (2017). A Large Kinome in a Large Cell: Stentor coeruleus Possesses Highly Expanded Kinase Families and Novel Domain Architectures. bioRxiv[Preprint] doi: 10.1101/16 8187

Robbins, J., Dilworth, S. M., Laskey, R. A., and Dingwall, C. (1991). Two interdependent basic domains in nucleoplasmin nuclear targeting sequence: identification of a class of bipartite nuclear targeting sequence. Cell 64, 615-623. doi: 10.1016/0092-8674(91)90245-T

Shang, Y., Song, X., Bowen, J., Corstanje, R., Gao, Y., Gaertig, J., et al. (2002). A robust inducible-repressible promoter greatly facilitates gene knockouts, conditional expression, and overexpression of homologous and heterologous genes in Tetrahymena thermophila. Proc. Natl. Acad. Sci. U.S.A. 99, 3734-3739. doi: 10.1073/pnas.052016199 
Togawa, K., Yan, Y. X., Inomoto, T., Slaugenhaupt, S., and Rustgi, A. K. (2000). Intestinal cell kinase (ICK) localizes to the crypt region and requires a dual phosphorylation site found in map kinases. J. Cell Physiol. 183, 129-139.

Vallesi, A., Di Pretoro, B., Ballarini, P., Apone, F., and Luporini, P. (2010). A novel protein kinase from the ciliate Euplotes raikovi with close structural identity to the mammalian intestinal and male-germ cell kinases: characterization and functional implications in the autocrine pheromone signaling loop. Protist 161, 250-263. doi: 10.1016/j.protis.2009. 12.002

van der Lee, R., Buljan, M., Lang, B., Weatheritt, R. J., Daughdrill, G. W., Dunker, A. K., et al. (2014). Classification of intrinsically disordered regions and proteins. Chem. Rev. 114, 6589-6631. doi: 10.1021/cr400 $525 \mathrm{~m}$
Conflict of Interest: The authors declare that the research was conducted in the absence of any commercial or financial relationships that could be construed as a potential conflict of interest.

The reviewer EV declared a past co-authorship with one of the authors AV to the handling Editor.

Copyright (c) 2019 Candelori, Yamamoto, Iwamoto, Montani, Amici and Vallesi. This is an open-access article distributed under the terms of the Creative Commons Attribution License (CC BY). The use, distribution or reproduction in other forums is permitted, provided the original author(s) and the copyright owner(s) are credited and that the original publication in this journal is cited, in accordance with accepted academic practice. No use, distribution or reproduction is permitted which does not comply with these terms. 